\title{
Objetos Virtuales de Aprendizaje: Una estrategia innovadora para la enseñanza de la Física
}

Virtual Learning Objects: An innovative strategy for teaching physics

\author{
Mery Carmita Fernández-Valverde \\ mery.fernandez@psg.ucacue.edu.ec \\ Universidad Católica de Cuenca, Azogues \\ Ecuador \\ https://orcid.org/0000-0002-7649-9130 \\ Darwin Gabriel Garcia-Herrera \\ dggarciah@ucacue.edu.ec \\ Universidad Católica de Cuenca, Azogues \\ Ecuador \\ https://orcid.org/0000-0001-6813-8100 \\ Cristián Andrés Erazo-Álvarez \\ cristianerazo@ucacue.edu.ec \\ Universidad Católica de Cuenca, Cuenca \\ Ecuador \\ https://orcid.org/0000-0001-8746-4788 \\ Juan Carlos Erazo-Álvarez \\ jcerazo@ucacue.edu.ec \\ Universidad Católica de Cuenca, Cuenca \\ Ecuador \\ https://orcid.org/0000-0001-6480-2270
}

Recibido: 28 de abril de 2020

Revisado: 15 de mayo de 2020

Aprobado: 10 de junio de 2020

Publicado: 29 de junio de 2020 


\title{
RESUMEN
}

La investigación tuvo por analizar los Objetos Virtuales de Aprendizaje dentro del proceso enseñanza - aprendizaje en la asignatura de Física en la búsqueda de la motivación de los estudiantes en su estudio. Fue de tipo descriptiva no experimental, de cohorte transversal, los datos se analizaron a través de técnicas estadísticas descriptivas y reflejan poca utilización de Objetos Virtuales de Aprendizaje para mejorar la comprensión de contenidos y la motivación, se evidencia también un alto grado de desconocimiento sobre este tema por parte de los docente, por lo que es necesario propender una capacitación al respecto, para su correcta aplicación y de esta manera, generar nuevos escenarios de aprendizajes significativos.

Descriptores: Educación; motivación; innovación pedagógica; aprendizaje activo. (Palabras tomadas del Tesauro UNESCO).

\begin{abstract}
The research had to analyze the Virtual Learning Objects within the teaching-learning process in the Physics subject in the search for the motivation of the students in their study. It was a non-experimental descriptive type, of cross-sectional cohort, the data was analyzed at Through descriptive statistical techniques and reflect little use of Virtual Learning Objects to improve the understanding of content and motivation, there is also a high degree of ignorance on this topic by teachers, so it is necessary to promote training to regarding, for its correct application and in this way, generate new significant learning scenarios.
\end{abstract}

Descriptors: Education; motivation; teaching method innovations; active learning. (Words taken from the UNESCO Thesaurus)

\section{INTRODUCCIÓN}

El propósito de la presente investigación es analizar la utilización de los Objetos Virtuales de Aprendizaje (OVA) como una nueva forma de impartir clases, para que de esta manera los estudiantes interactúen asertivamente en este proceso educativo, en este caso haciendo un realce en la asignatura de Física. Así pues, se obtendrán una gran diversidad de herramientas para mejorar el aprendizaje de los educandos, así por ejemplo se pueden utilizar imágenes, videos, audios, mapas mentales entre otros, creando innovaciones didácticas y ocasionando una gran importancia para que la enseñanza sea significativa y obtener mejores resultados.

Por otra parte, es muy importante hacer un breve análisis de cómo los estudiantes consideran a la asignatura de Física, e incluso se podría hablar de una parte afectiva, 
emocional y psicológica hacia la misma, pues bien es cierto que se le considera como una de las materias complicadas y de poco entendimiento e incluso de un limitada satisfacción y agrado, es ahí en donde se da la importancia de los OVA para modernizar los conocimientos y complacencia tanto de docentes como dicentes y obtener resultados mejores desde una nueva visión y perspectiva.

Cabe también recalcar que los estudiantes tienen un enfoque en los cuales piensan que esta asignatura solo se trata de resolución de problemas o ejercicios mediante la aplicación de modelos matemáticos sin considerar la gran importancia que tiene no solo para proseguir con sus estudios sino en el diario vivir de cada uno de nosotros. Cuando hablamos de aprendizaje esto no solo implica el alcanzar o dominar los mismo sino dominar habilidades como el escuchar, desarrollar y difundir mensajes pertinentes en diversos escenarios haciendo uso de los medios, códigos y herramientas apropiadas; la adecuada identificación y ordenamiento de las ideas le permite llegar a la información significativa donde los empleos de los OVA ayudaran de manera asertiva a solucionar determinada problemática establecida ya que cada día están tomando más protagonismo impactando en todo orden de actividades, y no se puede obviar la educación, desde las cotidianas hasta las más específicas y técnicas. Se torna entonces imperante el saber usarlas adecuadamente para poder así aprovecharlas en su máxima posibilidad, para que de esta manera obtener ventajas con respecto a las actividades que no cuentan con esta nueva forma de hacer las cosas. Esto implica una nueva oportunidad para renovar métodos antiguos de quehacer, representando un desafío importante para la sociedad actual.

Es por ello que el uso de los OVA en la actualidad se considera un instrumento eficaz, que nos ofrece una gran diversidad de provechos al momento de poner en práctica en el proceso enseñanza - aprendizaje. Se considera una necesidad la incorporación de las tecnologías en la educación, ya que son demasiadas las oportunidades que ofrece, desde ayudar en la motivación para el aprendizaje, hasta poder trabajar colaborativamente desde cualquier lugar.

Desafortunadamente existen asignaturas que están establecidas dentro de la malla curricular del Ministerio de Educación del Ecuador que no estamos dando la importancia que se debe, como es la Física. Esta es una materia muy necesaria 
dentro del proceso de aprendizaje e incluso para obtener su título de bachiller se debe rendir la prueba ser bachiller en la cual existen tópicos del dominio científico, misma que se incluye a la Física, sin embargo, la poca motivación de los estudiantes hace que fracase el proceso de enseñanza - aprendizaje. Es por ello que el objetivo de esta investigación es analizar los Objetos Virtuales de Aprendizaje dentro del proceso enseñanza - aprendizaje en la asignatura de Física en la búsqueda de la motivación de los estudiantes en su estudio.

\section{Referencial teórico}

La utilización de los OVA es una de las mejores combinaciones para obtener un contexto educativo y tecnológico eficaz (Callejas-Cuervo, Hernández-Niño \& PinzónVillamil, 2011); considerando como Objetos Virtuales de Aprendizaje (OVA) a cualquier recurso digital que pueda ser reutilizado para apoyar el aprendizaje. También se puede conceptualizar como el más pequeño de los elementos de información, comprensible y necesario para que de esta manera una persona pueda consegir un objetivo, una meta, resultado de aprendizaje o una destreza (Roig-Vila, Lledó-Carreres , \& Grau-Company , 2004). Si bien es cierto que no existe una definición unificada debido a que no se ha llegado a un concenso, sin embargo la idea general que engloba a los OVA es que son útiles para mejor aprendizajes mediante la tecnología (Callejas-Cuervo, Hernández-Niño , \& Pinzón-Villamil, 2011).

La educación está avanzando a pasos agigantados, todo esto en gran parte se debe al avance tecnológico que está dando cambios esenciales y fundamentales en la vida del ser humano, de ahí la necesidad de conocer más herramientas digitales fundamentales para un mejor desarrollo tanto de docentes como de estudiantes. $\mathrm{Si}$ bien es cierto como nos menciona (León, 2007) que el ser humano es diferente, es decir, es un mundo individual, es por ello que cada uno de nosotros aprendemos de forma diferente por la tanto la educación es un proceso que cada individuo va fortaleciendo con el pasar de los años, de ahí la necesidad de reforzar el aprendizaje que no es dado genéticamente al ser humano, lo que no es congénito, como el mismo nos dice eso es la educación. 
Los docentes debemos tener en cuenta que cada uno de los estudiantes tiene capacidades diferentes, por ende, sus ritmos de aprendizaje no son iguales es por ello el ingenio de utilizar los OVA adecuadamente garantizando un entendimiento colectivo. Teniendo en cuanta que los OVA pueden ser archivos o unidades digitales que proporcionan información con el beneficio que puedan ser utilizadas en una gran variedad de propuestas y contextos pedagógicos, garantizando una mejorara en el proceso de aprendizaje de los estudiantes, siendo un refuerzo para la educación (Latorre, 2018).

Los Objetos Virtuales de Aprendizaje contienen una variedad de recursos didácticos entre ellos tenemos los videos, audios, animaciones, documentos interactivos, mapas mentales, colecciones de imágenes los cuales transmiten conocimientos logrando un aprendizaje significativo, pudiendo estos ser utilizados de diferentes formas y sobre todo en cualquier dispositivos, de esta manera se facilita la utilización teniendo en cuenta que están en formatos diferentes para que puedan ser aprovechados por todos (Gomez, 2019).

Si bien es cierto la educación está atravesando por situaciones difíciles, los docentes como entes fundamentales dentro de este proceso deben estar preparados para todas estas dificultades que se van dando durante esta ardua y hermosa travesía, es por ello la necesidad de día a día superarse y actualizarse. Debido a esta dificultad (Morales-Martín, Gutiérrez-Mendoza, \& Ariza-Nieves, 2016) aporta con una guía sobre los OVA la cual sirve para que los docentes puedan orientarse de manera asertiva y eficaz, con estrategias metodológicas adecuadas y el proceso enseñanza aprendizaje sea lo más eficaz posible tanto para estudiantes como para los docentes. De esta manera se está motivando al estudiantado que se familiarice de una mejor manera con la física y lleguen a tener un cierto gusto por aprender esta asignatura y mejore sus conocimientos ante tal materia importante en el diario vivir.

Aunque existe un acceso fácil a internet por diferentes razones, tanto por su costo y su rápida accesibilidad, se ha estado impulsando constantemente a la utilización de las herramientas virtuales para la enseñanza sea la adecuada de esta manera estamos motivando constantemente a la juventud estudiantil (Cabrera-Medina, Sánchez-Medina, \& Rojas-Rojas, 2016). Una de las grandes ventajas que existen al 
aplicar los OVA es la diversidad de material de apoyo que se tiene de la misma manera la accesibilidad y flexibilidad al utilizar las estrategias metodológicas que más convenga de acuerdo a la temática impartida y afortunadamente sin costos Debido a que en los últimos años ha avanzado a pasos agigantados la tecnología, así mismo está sucediendo con los OVA, desafortunadamente el problema se ve inmerso es debido a que los docentes no aplicamos correctamente, previamente planificado, esto implica un reto para los docentes y aplicar estrategias metodológicas activas como manifiesta (Veytia, Lara Villanueva, \& García Robelo, 2018). Si bien es cierto en este trayecto educativo la buena utilización de estrategias metodológicas juega un papel muy importante para que resulte exitoso el aprendizaje de esta manera estamos garantizando el trabajo en equipo, el emprendimiento, el aprendizaje autónomo, la resolución de problema entre otros destacando el protagonismo de los estudiantes (García-Gajardo, Fonseca-Grandón, \& Concha-Gfell, 2015).

El docente como promotor de la educación es el que debe planificar adecuadamente sus clases, utilizando estrategias metodológicas activas, como es el caso del trabajo colaborativo en la cual sirve para que los estudiantes interactúen entre sí, compartan sus vivencias y saberes, de esta manera se retroalimenten con conocimientos nuevos enriquecidos que cuando se trabaja de una forma individual, pero el profesor debe garantizar que el ambiente dentro del aula sea el mejor, el más adecuado para que al trabajar colaborativamente se obtenga resultados fructíferos (Rodríguez-Zamora \& Espinoza-Núñez, 2017). De esta manera estamos dando una gran relevancia a los OVA debido a que se puede trabajar de una forma diferente aplicando esta estrategia metodológica como es el trabajo colaborativo y obteniendo resultados exitosos.

Debido a las grandes ventajas de los OVA y para tener una buena utilización (Pascuas-Rengifo, Jaramillo-Morales, \& Verástegui-González, 2015) en su artículo Desarrollo de objetos virtuales de aprendizaje como estrategia para fomentar la permanencia estudiantil en la educación superior nos da a conocer algunos componentes, especificaciones, en donde se alojan los OVA siendo estos: Pedagógico - comunicativo, el cual sirve para una mejor orientación tanto lo pedagógico, didáctico y diseño; Tecnológico que está dirigido en la aplicación de técnicas, tecnologías, que están encaminadas al diseño y recursos digitales; 
Estándares, especificaciones, es la aplicación de estándares relacionados con metadatos LOM CO, especificaciones SCORM, y políticas de derechos de autor para la publicación de recursos digitales como Creative Commons, Contenedor de los OVA que integra un servidor de aplicaciones con un esquema de administrador, herramientas, de comunicación, un contenedor de recursos digitales y control de acceso a usuarios.

Con la aplicación correcta de estos componentes se estaría obteniendo resultados muy favorables en beneficio de los educandos y de esta manera motivándolos a que si utilizamos recursos digitales se puede ver de otra perspectiva a la Física y llegar a tener empatía hacia esta materia y ver al gran significado que esta representa en nuestra vida.

Estas herramientas digitales y programas están ayudando de una manera tal que era imposible lograr de una forma tradicional, ya que se está consiguiendo que los estudiantes vayan construyendo sus propios conocimientos al ritmo de aprendizaje de ellos, obteniendo nuevas formas de evaluar siendo estas más completas, otras metodologías activando el conocimiento, haciendo más participativos y sobre todo que los Objetos Virtuales de Aprendizaje en su totalidad no tienen costo alguno y sirve para todo nivel educativo desde el más bajo hasta el más alto (Herrera-Cubides, Gelvez-García, \& Sánchez-Céspedes, 2014).

Debido a todos estos beneficios que nos ofrecen los OVA se puede mejorar el aprendizaje de los estudiantes llegando a la sensibilidad de cada uno de ellos y dándoles a conocer la importancia de la Física de allí nace la necesidad de analizar los Objetos Virtuales de Aprendizaje dentro del proceso enseñanza - aprendizaje en la asignatura de Física en la búsqueda de la motivación y compromiso de los estudiantes en su estudio, así pues lograremos tener un aprendizaje significativo y prepararles para un futuro mejor al servicio de la comunidad, determinando el grado de aceptación que tengan los estudiantes para que estos sean aplicados en sus clases. 


\section{MÉTODO}

La metodología que se utilizó para el análisis de los Objetos Virtuales de Aprendizaje es de carácter no experimental, pon cuanto no se estuvo directamente involucrada, siendo la misma cuantitativo, pues los datos recolectados miden características y valores numéricos, teniendo una cohorte transversal pues los datos fueron recolectados una sola vez (Bernal-Ávila, Erazo-Álvarez, \& Narváez-Zurita, 2019).

El estudio realizado se dio en la Unidad Educativa "Andrés F. Córdova" de la ciudad de Cañar en marzo del 2020, se contó con una muestra de 133 estudiantes mediante un muestreo aleatorio estratificado, 29 estudiantes que cursan el Primero de Bachillerato, 54 el Segundo de Bachillerato y 50 de Tercero de Bachillerato, la cual se realizó una encuesta mediante el aplicativo Google Drive empleando redes sociales, la misma que constaba de seis preguntas que midieron la comprensión de los contenidos estudiados en la asignatura de Física, la frecuencia de la utilización de los OVA por parte de los docentes, la frecuencia que les gustaría que sean utilizados los OVA en clases, motivación al recibir clases de Física, si les gustaría que se utilicen otros tipos de metodologías y que tipos de OVA es de su preferencia a ser utilizados para un mejor aprendizaje en esta asignatura.

La encuesta aplicada a los estudiantes fue una escala de Likert que constaba de 5 alternativas, así también 5 opciones de OVA para que sea selecciona el de mayor preferencia que les gustaría que fuese utilizados siendo estas: videos, documentos interactivos, mapas mentales, imágenes y audios. Para la verificación de la fiabilidad del instrumento de investigación se calculó el coeficiente Alfa de Cronbach obteniendo un resultado de 0,701 con 6 preguntas. En cuanto al análisis e interpretación de los datos se utilizó el software IBM SPSS Statistics 20 y un análisis descriptivo.

\section{RESULTADOS}

Continuando con el análisis de resultados para comprobar la veracidad o no de la hipótesis que si depende o no de la utilización de recursos digitales para que los estudiantes se motiven en clases de Física se utilizó la tabla de contingencia: estadísticos Chi cuadrado, para la cual se consideró dos variables, la independiente que es la frecuencia con que el docente utiliza recursos digitales para construir sus 
conocimientos y otra dependiente que es la frecuencia con que se sienten motivados los estudiantes al recibir clases de Física. Para la cual obtenemos los siguientes resultados:

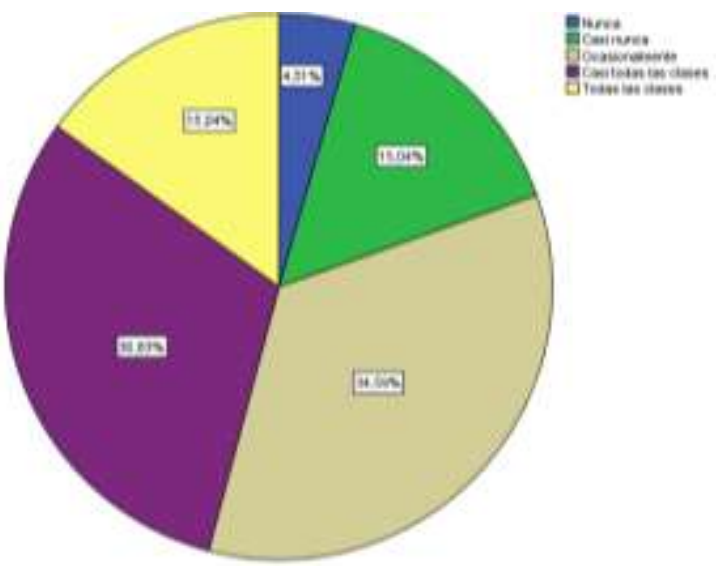

Figura 1. Utilización de recursos digitales. Fuente: Elaboración propia

El Figura 1 da a conocer que los docentes si utilizan recursos digitales para impartir clases teniendo una suma $80,46 \%$ desde ocasionalmente hasta todas las clases contra un 19,54 que es nunca y casi nunca.

\section{Tabla 1}

Utilización de recursos digitales y motivación

\begin{tabular}{lccc} 
& Valor & gl & Sig. asintótica (bilateral) \\
\hline Chi-cuadrado de Pearson & $32,085^{\mathrm{a}}$ & 16 & 0,010 \\
Razón de verosimilitudes & 31,954 & 16 & 0,010 \\
Asociación lineal por lineal & 18,854 & 1 & 0,000 \\
N de casos válidos & 133 & & \\
\hline
\end{tabular}

Fuente: Elaboración propia

Como se puede observar en la tabla 1 se interpreta que estas variables antes mencionas están estrechamente ligadas, es decir, que depende de la utilización de recursos digitales utilizados por los docentes para que los estudiantes se sientan motivados en las clases de Física y así poder obtener un mejor resultado de aprendizaje. Esto lo verificamos por cuanto los resultados de la Sig. asintótica 
(bilateral) es menor a 0,05.

De la misma manera continuamos con otra correlación que es entre los recursos utilizados por el docente y los contenidos comprendidos por los estudiantes en la asignatura de física, teniendo que:

\section{Tabla 2}

Utilización de recursos digitales y contenidos comprendidos

\begin{tabular}{lccc} 
& Valor & gl & Sig. asintótica (bilateral) \\
\hline Chi-cuadrado de Pearson & $37,736^{\mathrm{a}}$ & 16 & 0,002 \\
Razón de verosimilitudes & 41,908 & 16 & 0,000 \\
Asociación lineal por lineal & 26,627 & 1 & 0,000 \\
N de casos válidos & 133 & &
\end{tabular}

Fuente: Elaboración propia

Como se puede observar en la tabla 2 que Sig. asintótica (bilateral) es menor a 0,05 se estaría afirmando que los recursos digitales son un factor muy importante en el proceso de aprendizaje que ya depende para que los contenidos estudiados sean comprendidos y no queden flotando.

Ahora bien, es muy importante conocer qué tipo de OVA puede ser es más conveniente y sobre todo el que más les llame la atención a los estudiantes y se sientan motivados al recibir clases de Física, esta pregunta nos contestamos observando la tabla 3.

\section{Tabla 3}

Tipos de Objetos Virtuales de Aprendizaje (OVA) utilizados preferentemente

\begin{tabular}{llcccc} 
& Frecuencia & Porcentaje & $\begin{array}{c}\text { Porcentaje } \\
\text { válido }\end{array}$ & $\begin{array}{c}\text { Porcentaje } \\
\text { acumulado }\end{array}$ \\
\hline \multirow{2}{*}{ Válidos } & Videos & 94 & 70,7 & 70,7 & 70,7 \\
& Documentos interactivos & 14 & 10,5 & 10,5 & 81,2 \\
& Mapas mentales & 11 & 8,3 & 8,3 & 89,5 \\
& Imágenes & 9 & 6,8 & 6,8 & 96,2 \\
& Audios & 5 & 3,8 & 3,8 & 100,0 \\
& Total & 133 & 100,0 & 100,0 &
\end{tabular}

Fuente: Elaboración propia 
Uno de los OVA que más les interesa a los estudiantes son los videos con un porcentaje muy alto como es un $70,7 \%$ con una frecuencia de 94 estudiantes de 133 , los otros OVA son de su interés, pero no son prioritarios, pues sus porcentajes son muy bajos.

Los recursos digitales entre ellos los OVA son una gran ventaja en el proceso de aprendizaje. Los educandos establecen una gran oportunidad de utilizar los OVA y de esta manera desarrollar sus habilidades y capacidades a su máximo potencial, trabajando en forma colectiva o individual, pero para poder tener mejores resultados y ellos se motiven para utilizar recursos didácticos se debe trabajar a tempranas edades, después a los estudiantes ya no les interesa aprender de una forma diferente puesto que se acostumbraron a ese ritmo de aprendizaje (Rodríguez-Zamora y Espinoza-Núñez, 2017).

Afortunadamente el internet tiene un gran beneficio que es el proporcionar información de todo tipo, siendo una herramienta de búsqueda muy importante, una de las ventajas es que los estudiantes utilizan para reforzar algunos contenidos que no fueron comprendidos en su totalidad de esta manera ellos refuerzan sus conocimientos para mejorar en su rendimiento académico, los estudiantes manifiestan que acuden al internet para fines educativos siendo los OVA benefactores para la obtención de esta información con sus múltiples productos digitales (Feria-Marrugo \& Zúñiga, 2016).

Por otro lado (Morales, Mazzitelli, \& del Carmen Olivera, 2015) nos comparte su investigación en la cual los estudiantes aluden que las asignatura de Física y Química les llama la atención, esto implica a que los docentes debemos prepararnos cada día más, utilizar estrategias metodológicas activas, para obtener toda la capacidad de los estudiantes. Es por ello que para el aprendizaje de la Física sea más eficaz es vital que se realicen actividades prácticas, para ello se puede utilizar una gran diversidad de material didácticos ya sean dispositivos elaborados por empresas o simplemente materiales que se utilizan cotidianamente, con esta forma de trabajar se facilita la comprensión de contenidos e incluso una buena opción es la utilización de recursos digitales en este caso uno de los OVA (García-Molina, 2015). 


\section{PROPUESTA}

El modelo de la utilización de los objetos Virtuales de Aprendizaje se efectuó con un enfoque en cuatro aspectos claramente definidos: planificar, ejecutar, controlar y actuar, con la finalidad de mejorar el proceso enseñanza - aprendizaje en la asignatura de Física, así como también motivar a los estudiantes por la afinidad hacia esta asignatura (Figura 2). De tal manera que los resultados sean diferentes y de esta manera obtener un aprendizaje significativo durante todo su proceso.

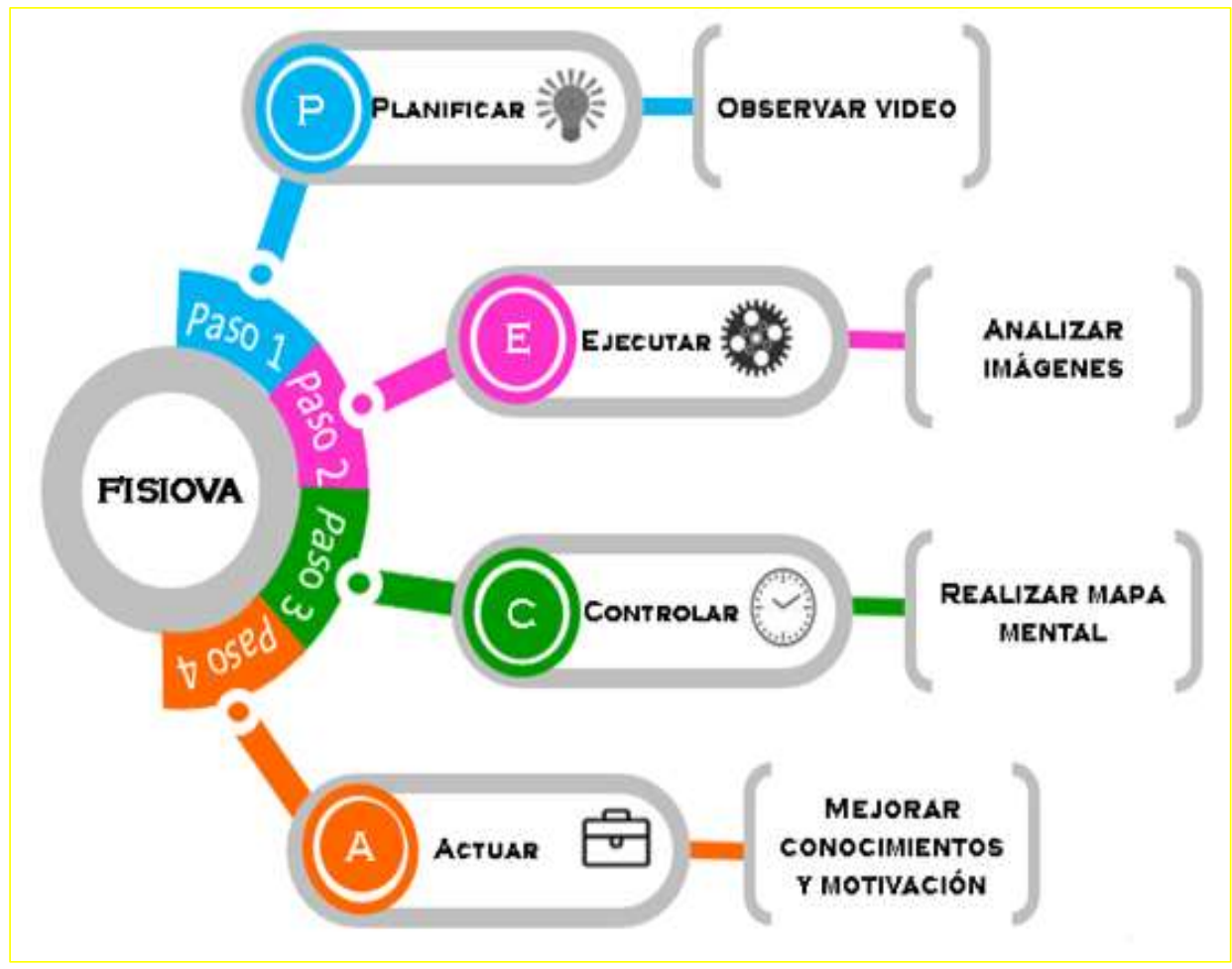

Figura 1. Modelo de propuesta de Objetos Virtuales de Aprendizaje. Fuente: Elaboración propia

A continuación, se detalla cada uno de sus principales componentes:

Planificar: en este aspecto se parte con una introducción que en este caso viene a ser la anticipación de una clase, es por ello que se procede en primer lugar a desarrollar la Destreza con Criterio de Desempeño (DCCD) y el Objetivo con el que se va a trabajar en la hora clase. En base a estos dos aspectos se procede a proyectar 
un video de una duración aproximada de unos tres a 4 minutos, este video debe estar estrechamente entrelazado tanto con la DCCD y el Objetivo de la clase, en este caso si hablamos de un tema en específico que es el fenómeno de la fuerza el video debe ser claro y conciso en donde intervenga este fenómeno, para continuar con una estrategia metodológica que en este caso sería la lluvia de ideas, para que los estudiantes reflexionen, analicen y obtengan sus ideas principales del mismo y compartan sus opiniones entre todos los estudiantes, para finalizar con una idea general y culminar exitosamente con esta etapa de la anticipación, generando interés, motivación y conocimientos significativos.

Ejecutar: en este aspecto se continua con la segunda etapa de una clase, que es la construcción, es aquí en donde los estudiantes van a adquirir sus conocimientos los mismos que deben ser comprendidos en su totalidad de una manera eficaz, pero sobre todo que ellos se sientan motivados y la clase sea una diversión más no una exigencia, es por ello que se presentan diversas imágenes o gráficos los cuales deben contener ecuaciones del tema tratado, la parte teórica, ejercicios modelos y fenómenos de la vida diaria en la cual este inmerso, como este caso estamos hablando de la fuerza, las imágenes deben ser de cuerpos que desarrollen fuerzas en diferentes situaciones, las mismas que deben ser claras para que los estudiantes puedan observar y diferenciar cada uno de las aspectos de las imágenes.

Controlar: esta es la etapa final de una clase, la consolidación, es aquí en donde se verifica la comprensión de los conocimientos adquiridos, verificando la eficacia del desarrollo de la misma. En esta parte obtenemos los resultados mediante una evaluación la cual consta de una realización o elaboración de un mapa mental en la cual sea especificado las características principales del fenómeno de la fuerza, con sus respectivas ecuaciones. Para la cual se forman grupos o equipos de trabajo mediante la estrategia metodológica que es el trabajo colaborativo. En esta parte también se desarrolla ejercicios de aplicación, para la cual los estudiantes deben tener todos los conocimientos necesarios para poder desarrollar estas actividades.

Actuar: este es el aspecto final de la propuesta, es aquí en donde se van a ver los resultados finales, en este caso los estudiantes obtendrán mejores resultados, siendo estos la motivación y la comprensión del tema, es decir que las utilizaciones de los 
OVA son de gran ayuda e importancia en el proceso de enseñanza - aprendizaje y motivación, debido a que se está modificando la forma de impartir clases, realizando una innovación pedagógica.

Es muy importante tener en cuenta que los OVA pueden ser utilizados en diferentes asignaturas y contenidos, solo depende de cada uno de nosotros como docentes de ver la mejor manera y forma de utilizar para que los resultados sean fructíferos y se obtenga resultados exitosos, solo depende de nosotros hacer la diferencia.

\section{CONCLUSIONES}

Como resultado se puede decir que las metodologías activas y recursos didácticos que utilice el docente es un papel fundamental e importante para que los estudiantes se motiven al recibir clases en la asignatura de Física, es por ello la necesidad de la actualización docente sobre todo en el ámbito tecnológico, por ende, en los OVA para que sean estos utilizados adecuadamente y con mayor frecuencia.

Otro factor muy importante que conlleva la utilización de los recursos didácticos son los contenidos estudiados, desafortunadamente no todos los temas analizados en clases representan algo significativo para los estudiantes, es por ello que se debe saber que OVA se debe utilizar y en qué momento anteriormente planificada por el docente para que no resulte un fracaso la utilización de los mismos. Como se pudo observar que los docentes se utilizan recursos digitales en las clases, lo que hace falta es saber cómo se debe utilizar correctamente y que estrategia metodológica se debe utilizar es por ello que sería bueno realizar una capacitación sobre la utilización correcta de los OVA.

Para concluir con esta investigación se consideró la opinión de los estudiantes en cuanto a los tipos de Objetos Virtuales de Aprendizajes que es de su preferencia siendo estos los videos, ya que cuando se observa se tiene mayor facilidad de aprendizaje y entendimiento, siempre y cuando estos estén relacionados con la temática a tratar y sobre todo con la asignatura de Física.

Definitivamente los OVA es una innovación, una nueva forma de ver la docencia aprendizaje - estudiante no solo en la asignatura de Física, estos se pueden utilizar en todas las materias y todas las edades, siempre y cuando estén correctamente 
planificadas y utilizadas, existe una gran diversidad para que se pueda elegir la más conveniente de acuerdo al tema que se está tratando, solo depende de nosotros los docentes cambiar la forma de ver las cosas para obtener mejores resultados en la educación, pues esto es lo que nos va a llegar a la gloria y así poder servir a la sociedad en beneficio de la misma.

\section{FINANCIAMIENTO}

No monetario.

\section{AGRADECIMIENTO}

Al personal Directivo y Docente la Unidad Educativa "Andrés F. Córdova" de la ciudad de Cañar.

\section{REFERENCIAS CONSULTADAS}

Bernal-Ávila, E. M., Erazo-Álvarez, J. C., \& Narváez-Zurita, C. I. (2019). Estructuras organizativas favorables a la Eficiencia Empresarial. [Organizational structures favorable to Business Efficiency]. Revista Arbitrada Interdisciplinaria Koinonía, 3-31. http://dx.doi.org/10.35381/r.k.v4i1.370

Cabrera-Medina, J. M., Sánchez-Medina, I. I., \& Rojas-Rojas, F. (2016). Uso de objetos virtuales de aprendizaje OVAS como estrategia deenseñanza aprendizaje inclusivo y complementario a los cursos teóricos - prácticos. Una experiencia con estudiantes del curso física de ondas [Use of OVAS virtual learning objects as a teac]. Revista educación en ingeniería, 11(22), 4.12. Obtenido de https://n9.cl/9duf

Callejas-Cuervo, M., Hernández-Niño , E. J., \& Pinzón-Villamil, J. N. (2011). Objetos de aprendizaje, un estado del arte [Learning objects, a state of the art]. Entramado, 71, 176-189. Obtenido de https://n9.cl/sxiu

Feria-Marrugo, I. M., \& Zúñiga, K. S. (2016). Objetos virtuales de aprendizaje y el desarrollo de aprendizaje autónomo en el área de inglés [Virtual learning objects and the development of autonomous learning in the English]. Revista Praxis, 12, 63-77. doi:http://dx.doi.org/10.21676/23897856.1848 
García-Gajardo, F., Fonseca-Grandón, G., \& Concha-Gfell, L. (2015). Aprendizaje y rendimiento académico en educación superior: un estudio comparado [Academic learning and performance in higher education: a comparative study]. Revista Electrónica "Actualidades Investigativas en Educación", 15(3), 1-25. https://dx.doi.org/10.15517/aie.v15i3.21072

García-Molina, R. (2015). Experimentos de Física usando las TIC y elementos de bajo costo [Physics experiments using ICT and low cost elements]. Revista Eureka sobre Enseñanza y Divulgación de las Ciencias, 2(1), 231-232. Recuperado de https://n9.cl/7vgc

Gomez, M. M. (2019). Elearningmasters. Recuperado de https://n9.cl/e0i3

Herrera-Cubides, J. F., Gelvez-García, N. Y., \& Sánchez-Céspedes, J. M. (2014). Standardization initiatives in the production of virtual learning objects. JISTEM J.Inf.Syst. Technol. Manag, 11(1) https://doi.org/10.4301/S180717752014000300009

Latorre, C. F. (2018). Objetos virtuales de aprendizaje. Diseño de ambientes educativos basados en NTIC [Design of educational environments based on NTIC]. Recuperado de https://n9.cl/PpFx

León, A. (2007). Qué es la educación [What is education]. Educere, 11(39), 595-604. Obtenido de https://www.redalyc.org/articulo.oa?id=35603903

Morales, L. M., Mazzitelli, C. A., \& del Carmen Olivera, A. (2015). La enseñanza y el aprendizaje de la Física y de la Química en el nivel secundario desde la opinión de estudiantes [The teaching and learning of Physics and Chemistry at the secondary level from the opinion of students]. Revista Electrónica de Investigación en Educación en Ciencias, 10(2), 11-20. Recuperado de https://n9.cl/02w3x

Morales-Martín, L. Y., Gutiérrez-Mendoza, L., \& Ariza-Nieves, L. M. (2016). Guía para el diseño de objetos virtuales de aprendizaje(OVA). Aplicación al proceso enseñanza-aprendizajedel área bajo la curva de cálculo integral [Guide for the design of virtual learning objects (OVA). Application to the teaching-learning process of t]. Revista Científica General José María Córdova, 127-147. Recuperado de https://n9.cl/9q9k

Pascuas-Rengifo, Y. S., Jaramillo-Morales, C. O., \& Verástegui-González, F. A. (2015). Desarrollo de objetos virtuales de aprendizaje como estrategia para fomentar la permanencia estudiantil en la educación superior [Development of virtual learning objects as a strategy to promote student permanence in higher education]. Revista Escuela de Administración de Negocios(79), 116-129. Recuperado de https://www.redalyc.org/pdf/206/20643042008 
Rodríguez-Zamora, R., \& Espinoza-Núñez, L. A. (2017). Trabajo colaborativo y estrategias de aprendizaje en entornos virtuales en jóvenes universitarios [Collaborative work and learning strategies in virtual environments in young university students]. RIDE Revista Iberoamericana para la Investigación y el Desarrollo Educativo, 7(14), 1-24.https://dx.doi.org/10.23913/ride.v7i14.274

Roig-Vila, R., Lledó-Carreres , A., \& Grau-Company , S. (2004). Objetos d aprendizaje (Learning objects) como respuesta educativa al alumnadocon altas capacidades desde la inclusión digital [Learning objects as an educational response to students with high abilities from digital inclusion]. Universidad de Alecante. Recuperado de http://hdl.handle.net/10045/4641

Veytia, M. G., Lara Villanueva, R. S., \& García Robelo, O. (2018). Objetos Virtuales de Aprendizaje en la Educación Superior [Virtual Objects of Learning in Higher Education]. Eikasia. Recuperado de https://n9.cl/660b 